\title{
Sur le graphe divisoriel
}

\author{
par \\ Paul Erdős (Budapest) et Eric SaIAs (Paris)
}

1. Introduction. On définit sur l'ensemble des entiers positifs $\leq x$ les deux relations $\mathcal{R}_{f}$ et $\mathcal{R}_{g}$ suivantes :

$$
\begin{gathered}
a \mathcal{R}_{f} b \quad \text { si et seulement si } a \text { divise } b \text { ou } b \text { divise } a ; \\
a \mathcal{R}_{g} b \text { si et seulement si } \operatorname{ppcm}(a, b) \leq x .
\end{gathered}
$$

On remarque que

$$
\text { si } a \mathcal{R}_{f} b \text { alors } a \mathcal{R}_{g} b .
$$

On appelle graphe divisoriel le graphe de la relation $\mathcal{R}_{f}$. Enfin, on appelle chaîne d'entiers $\leq x$ de longueur $l$, pour la relation $\mathcal{R}_{f}$ (respectivement $\left.\mathcal{R}_{g}\right)$, toute suite $\left(n_{i}\right)_{1 \leq i \leq l}$ d'entiers $\leq x$, deux à deux distincts, et vérifiant pour tout $i(1 \leq i<l) n_{i} \mathcal{R}_{f} n_{i+1}\left(\right.$ resp. $\left.n_{i} \mathcal{R}_{g} n_{i+1}\right)$.

L'étude asymptotique (quand $x$ tend vers $+\infty$ ) du graphe divisoriel (et du graphe de $\mathcal{R}_{g}$ ) se subdivise en plusieurs problèmes. L'un de ceux-ci consiste à estimer la longueur maximale $f(x)$ (resp. $g(x)$ ) d'une chaîne d'entiers $\leq x$ pour la relation $\mathcal{R}_{f}$ (resp. $\mathcal{R}_{g}$ ). Les travaux [1], [3], [4], [5] et [7] traitent de cette question. On sait maintenant [5] qu'il existe deux constantes strictement positives $c$ et $c^{\prime}$ telles que pour tout $x \geq 2$, on ait

$$
c \frac{x}{\log x} \leq f(x) \leq g(x) \leq c^{\prime} \frac{x}{\log x} .
$$

Un autre problème est d'estimer le nombre minimum de chaînes d'entiers $\leq x$ nécessaires pour recouvrir tous les entiers $\leq x$. C'est cette question qui fait l'objet du présent travail.

En fait, il y a deux questions suivant que l'on autorise ou non aux chaînes d'avoir des entiers en commun. On désigne par $\varphi(x)$ (resp. $\gamma(x))$ le nombre minimum de chaînes $\mathcal{C}_{i}(1 \leq i \leq \varphi(x))$ (resp. $\left.1 \leq i \leq \gamma(x)\right)$ d'entiers $\leq x$ pour la relation $\mathcal{R}_{f}$ (resp. $\mathcal{R}_{g}$ ) telles que tout entier $\leq x$ apparait au moins dans une de ces chaînes. Dans le cas où on oblige les chaînes $\mathcal{C}_{i}$ à être deux à deux disjointes, on désigne par $\varphi^{*}(x)$ et $\gamma^{*}(x)$ les quantités 
correspondant respectivement à $\varphi(x)$ et $\gamma(x)$. Nous pouvons maintenant énoncer nos résultats.

ThÉORÈme 1. Il existe deux constantes strictement positives $c_{1}$ et $c_{2}$ telles que pour tout $x \geq 2$, on ait

$$
c_{1} \frac{x}{\log x} \leq \gamma(x) \leq \varphi(x) \leq c_{2} \frac{x}{\log x} .
$$

ThÉORÈME 2. Il existe une constante strictement positive $c_{3}$ telle que pour tout $x \geq 2$, on ait

$$
c_{3} x \leq \gamma^{*}(x) \leq \varphi^{*}(x) \leq x / 2 .
$$

2. Notations. On désigne par $\Omega(n)$ le nombre de facteurs premiers de l'entier $n$, comptés avec leur multiplicité.

On désigne par $P(n)$ (respectivement $\left.P^{-}(n)\right)$ le plus grand (resp. petit) facteur premier de l'entier $n>1$. On pose de plus $P(1)=1$ et $P^{-}(1)=\infty$.

On désigne usuellement par $\Psi(x, y)$ et $\Phi(x, z)$ les fonctions

$$
\Psi(x, y)=\operatorname{card}\{n \leq x: P(n) \leq y\}
$$

et

$$
\Phi(x, z)=\operatorname{card}\left\{n \leq x: P^{-}(n)>z\right\} .
$$

Les lettres $p$ et $q$ désigneront des nombres premiers génériques.

On désignera par $n_{1}-n_{2}-\ldots-n_{l}$ la suite finie d'entiers $\mathcal{S}=\left(n_{i}\right)_{1 \leq i \leq l}$. Si $\mathcal{S}_{1}=\left(n_{i}\right)_{1 \leq i \leq l}$ et $\mathcal{S}_{2}=\left(m_{j}\right)_{1 \leq j \leq k}$ sont deux suites finies d'entiers, on désignera par $\mathcal{S}_{1}-\mathcal{S}_{2}$ la suite $n_{1}-n_{2}-\ldots-n_{l}-m_{1}-m_{2}-\ldots-m_{k}$. On sera aussi amenés à concaténer des suites d'entiers et des entiers. Par exemple, si $a$ est un entier, on désignera par $\mathcal{S}-a$ la suite d'entiers $n_{1}-n_{2}-\ldots-n_{l}-a$.

\section{Lemmes généraux}

LEMME 1 . On a pour $x \geq 2 z \geq 4$,

$$
\Phi(x, z)=x \prod_{p \leq z}\left(1-\frac{1}{p}\right)\left(1+O\left(x^{-1 /(3 \log z)}\right)\right) .
$$

Lemme 2 . On a pour $x \geq y \geq 2$,

$$
\Psi(x, y) \ll x^{1-1 /(2 \log y)} .
$$

Lemme 3. Il existe une constante $x_{0}$ telle que pour $x \geq x_{0}$, il $y$ ait toujours au moins un nombre premier dans l'intervalle $\left[x, x+x^{2 / 3}[\right.$.

Le Lemme 1 découle facilement des corollaires III.6.7.3 et III.6.3.1 de [6]. Le Lemme 2 apparaît au Théorème III.5.1 de [6]. Enfin, on peut consulter par exemple [2] (Chapitre 28) pour une démonstration du Lemme 3. En 
fait, seul l'existence d'un réel $\theta<1$ tel que pour tout $x$ suffisamment grand, l'intervalle $\left[x, x+x^{\theta}[\right.$ contient au moins un nombre premier, nous est utile ici.

4. Démonstration du Théorème 2. Montrons que $\varphi^{*}(x) \leq x / 2$. A tout nombre impair $n$ inférieur ou égal à $x$, on associe la chaîne notée $\mathcal{C}_{n}: n-2 n-4 n-\ldots-2^{\alpha} n$ où $2^{\alpha}$ est la plus grande puissance de 2 telle que $2^{\alpha} n \leq x$. Pour $2 \leq x<3$, la chaîne $\mathcal{C}_{1}$ convient. Pour $x \geq 3$, on considère la famille de chaînes constituée des $\mathcal{C}_{n}$ avec $n$ impair $\geq 5$ et de la chaîne $\mathcal{C}_{3}-\mathcal{C}_{1}$. On obtient ainsi une famille d'au plus $x / 2$ chaînes deux à deux disjointes, qui recouvrent tous les entiers $\leq x$.

L'inégalité $\gamma^{*}(x) \leq \varphi^{*}(x)$ résulte de $(1)$.

Montrons maintenant que $\gamma^{*}(x) \gg x$. Cette inégalité est triviale pour $x$ borné. On peut donc supposer $x$ suffisamment grand. Soit $\left(\mathcal{C}_{j}\right)_{1 \leq j \leq k(x)} k(x)$ chaînes d'entiers $\leq x$, au sens de $\mathcal{R}_{g}$, deux à deux disjointes, et qui recouvrent tous les entiers $\leq x$. Soit $z$ un paramètre que l'on fixera ultérieurement. Soit $\mathcal{N}_{x, z}$ l'ensemble des entiers $n$ qui vérifient les deux propriétés suivantes :

$$
x / 2<n \leq x \text { et } P^{-}(n)>z,
$$

(3) la chaîne $\mathcal{C}_{j}$ qui contient $n$ n'est pas réduite au seul entier $n$.

D'après (3), chaque entier $n$ de $\mathcal{N}_{x, z}$ admet au moins un voisin $v$ dans sa chaîne, tel que $\operatorname{ppcm}(n, v) \leq x$. D'après $(2)$, on en déduit que $v<x / z$. On construit ainsi une application

$$
V: \mathcal{N}_{x, z} \rightarrow \mathcal{V}_{x, z}=\{v: v<x / z\} .
$$

Comme les chaînes $\left(\mathcal{C}_{j}\right)_{1 \leq j \leq k(x)}$ sont deux à deux disjointes, chaque entier de $\mathcal{V}_{x, z}$ est l'image d'au plus deux entiers de $\mathcal{N}_{x, z}$ par l'application $V$. Donc

$$
\operatorname{card} \mathcal{N}_{x, z}<2 x / z \text {. }
$$

Par ailleurs, d'après le Lemme 1 et la formule de Mertens, on a pour $2 \leq$ $z \leq \log x$ et $x$ suffisamment grand

$$
\operatorname{card}\left\{x / 2<n \leq x: P^{-}(n)>z\right\} \gg x / \log z .
$$

On peut donc choisir $z_{0}$ tel que pour $x$ suffisamment grand, on ait

$$
\operatorname{card} \mathcal{N}_{x, z_{0}} \leq \frac{1}{2} \operatorname{card}\left\{x / 2<n \leq x: P^{-}(n)>z_{0}\right\} .
$$

Donc au moins la moitié des entiers $n$ vérifiant (2) avec $z=z_{0}$ appartiennent à des chaînes réduites à un entier. Comme card $\left\{x / 2<n \leq x: P^{-}(n)>\right.$ $\left.z_{0}\right\} \asymp x$ pour $x$ suffisamment grand, cela montre que le nombre $k(x)$ de chaînes vérifie $k(x) \gg x$. Cela achève la démonstration du Théorème 2 .

5. Construction de chaînes à extrémités fixées et passant par un entier donné. Les lemmes de ce paragraphe sont élémentaires et faciles 
à démontrer. Dorénavant, quand on écrira "chaîne d'entiers", on entendra implicitement "chaîne d'entiers au sens de $\mathcal{R}_{f}$ ". Nous appellerons de plus suite d'entiers en relation toute suite $\left(n_{i}\right)_{1<i<l}$ d'entiers vérifiant pour tout $i(1 \leq i<l), n_{i} \mathcal{R}_{f} n_{i+1}$. Les entiers $n_{1}$ et $n_{l}$ seront appelés extrémités de la suite $\left(n_{i}\right)_{1 \leq i \leq l}$.

LEMme 4. De toute suite $\mathcal{S}$ d'entiers en relation, on peut extraire une chaîne qui a les mêmes extrémités que $\mathcal{S}$.

Démonstration. Soit $\mathcal{S}=\left(n_{i}\right)_{1 \leq i \leq l}$ une suite d'entiers en relation. Si $n_{i}=n_{j}$ pour $1 \leq i<j \leq l$ la suite extraite

$$
n_{1}-n_{2}-\ldots-n_{i}-n_{j+1}-n_{j+2}-\ldots-n_{l}
$$

est encore une suite d'entiers en relation qui a les mêmes extrémités que la suite initiale. En itérant, si besoin est, cette opération, on obtient en un nombre fini d'étapes une suite extraite de $\mathcal{S}$ qui est une chaîne et qui a les mêmes extrémités que $\mathcal{S}$.

LEMME 5. Soit $x$ un réel $\geq 2$. Soit a et $n$ deux entiers vérifiant $2 \leq a \leq x$, a est pair, $2 \leq n \leq x, P(n) \leq x / 2$ et $P(a) \leq P(n)$. Alors il existe une chaîne $\mathcal{C}$ d'entiers $\leq x$ vérifiant les propriétés suivantes:

- le premier élément de $\mathcal{C}$ est a,

- le dernier élément de $\mathcal{C}$ est 1 ,

- l'entier $n$ est un élément de $\mathcal{C}$,

- tous les entiers $m$ de $\mathcal{C}$ vérifient $P(m) \leq P(n)$.

Démonstration. La suite $a-2-2 P(n)-P(n)-n-1$ est une suite d'entiers $\leq x$ en relation, qui contient $n$ et dont les extrémités sont $a$ et 1 . A partir de cette suite, on obtient une chaîne convenable en appliquant, si besoin est, le Lemme 4.

Lemme 6. Soit $x$ un réel $\geq 2$. Soit $n$ un entier positif vérifiant $2 \leq n \leq x$ si $\Omega(n) \neq 2$, ou $n \leq x / 2$ si $\Omega(n)=2$. Soient $a$ et $b$ deux entiers distincts vérifiant $\Omega(a) \geq 3, a P(n) \leq x / 2, P(a)<P(n)$ et de manière symétrique $\Omega(b) \geq 3, b P(n) \leq x / 2, P(b)<P(n)$. Alors il existe une chaîne $\mathcal{C}$ d'entiers $\leq x$ vérifiant les propriétés suivantes:

- les extrémités de $\mathcal{C}$ sont les entiers a et $b$,

- l'entier $n$ est un élément de $\mathcal{C}$,

- à l'exception de a et $b$, tous les entiers de $\mathcal{C}$ sont divisibles par $P(n)$.

Démonstration. Posons $p=P(n)$. Si $n=p$, la suite $a-a p-p-b p-b$ est une chaîne convenable.

On suppose dorénavant $\Omega(n) \geq 2$ et on désigne par $q$ un nombre premier tel que $p q$ divise $n$. Comme $a$ et $b$ jouent des rôles indifférenciés, on peut 
supposer également $a<b$. En utilisant les hypothèses faites sur $x, n, a$ et $b$, on vérifie aisément que la suite

$$
a-a p-p-n-q p-2 q p-2 p-2 b p-b
$$

est une suite d'entiers $\leq x$ en relation avec

$$
\{a, a p, p\} \cap\{q p, 2 q p, 2 p, 2 b p, b\}=\emptyset .
$$

En utilisant, si besoin est, la procédure décrite dans la démonstration du Lemme 4 , on obtient, à partir de cette suite, une chaîne $\mathcal{C}$ ayant les propriétés requises.

6. Démonstration du Théorème 1. L'inégalité $\gamma(x) \leq \varphi(x)$ résulte de (1) et la minoration $\gamma(x) \gg x / \log x$ est facile. En effet, pour une famille de chaînes $\leq x$, au sens de $\mathcal{R}_{g}$, qui recouvre tous les entiers $\leq x$, les nombres premiers $p$ tels que $x / 2<p \leq x$ ont pour seul voisin éventuel l'entier 1 . Donc dans une chaîne donnée, il y a au plus deux tels nombres premiers. L'estimation asymptotique

$$
\pi(x)-\pi(x / 2) \asymp x / \log x
$$

permet alors de conclure.

Pour démontrer la majoration $\varphi(x) \ll x / \log x$, on partitionne les entiers $n \leq x$ en quatre ensembles. Pour chacun de ces ensembles $E$, on montre dans l'un des lemmes suivants que l'on peut recouvrir les entiers de $E$ avec $O(x / \log x)$ chaînes d'entiers $\leq x$. Pour simplifier les notations, on représentera toujours dans les lemmes suivants par $\left(\mathcal{C}_{r}\right)$ la famille de chaînes qui recouvre l'ensemble $E$, bien que cette famille de chaînes varie avec $E$. On fera de même avec la quantité $R(x)$.

Lemme 7. Soit $x \geq 2$. Il existe une famille $\left(\mathcal{C}_{r}\right)_{0 \leq r \leq R(x)}$ de $R(x)+1$ chaînes d'entiers $\leq x$ avec

$$
R(x) \ll x \exp \{-\sqrt{2 \log x}\}
$$

telle que

(4) tout entier positif $n \leq x$ avec $P(n) \leq \sqrt{x / 2}$ est élément d'au moins l'une des $R(x)+1$ chaînes $\mathcal{C}_{r}$.

LEMME 8. Il existe un entier $\alpha_{0}$ tel que pour $x \geq 2$, il existe une famille $\left(\mathcal{C}_{r}\right)_{1 \leq r \leq R(x)}$ de $R(x)$ chaînes d'entiers $\leq x$ avec

$$
R(x) \ll x / \log x
$$

telle que tout entier $n$ vérifiant

$$
\sqrt{x / 2}<P(n) \leq x / 2^{\alpha_{0}}
$$


et

$$
\begin{array}{ll}
2 \leq n \leq x & \text { si } \Omega(n) \neq 2, \\
n \leq x / 2 & \text { si } \Omega(n)=2
\end{array}
$$

est élément d'au moins l'une des $R(x)$ chaînes $\mathcal{C}_{r}$.

Lemme 9. Soit $\alpha_{0}$ un entier fixé. Soit $x \geq 2$. Il existe une famille $\left(\mathcal{C}_{r}\right)_{1 \leq r \leq R(x)}$ de $R(x)$ chaînes d'entiers $\leq x$ avec

$$
R(x) \ll x / \log x
$$

telle que tout entier positif $n \leq x$ vérifiant $P(n)>x / 2^{\alpha_{0}}$ est élément d'au moins l'une des $R(x)$ chaînes $\mathcal{C}_{r}$.

Lemme 10. Soit $x \geq 2$. Il existe une famille $\left(\mathcal{C}_{r}\right)_{1 \leq r \leq R(x)}$ de $R(x)$ chaînes d'entiers $\leq x$ avec

$$
R(x) \ll x / \log x
$$

telle que tout entier positif $n$ vérifiant $x / 2<n \leq x$ et $\Omega(n)=2$ est élément d'au moins l'une des $R(x)$ chaînes $\mathcal{C}_{r}$.

On adopte les notations suivantes : $2=p_{1}<p_{2}<\ldots$ désigne la suite croissante des nombres premiers. De plus, $\mathcal{D}(X, Y)$ désigne une chaîne générique d'entiers $n$ vérifiant $n \leq X$ et $P(n) \leq Y$. Enfin, $p \mathcal{D}(X, Y)$ désigne la chaîne obtenue en multipliant chaque entier de la chaîne $\mathcal{D}(X, Y)$ par le nombre premier $p$.

Les résultats étant triviaux pour $x$ borné, on pourra toujours supposer $x$ suffisamment grand.

Démonstration $\mathrm{du}$ Lemme 7 . On désigne par $\mathcal{C}_{0}$ une chaîne formée de toutes les puissances de 2 (y compris l'entier 1), inférieures ou égales à $x$, prises dans un ordre quelconque.

On suppose construites les chaînes $\mathcal{C}_{0}, \mathcal{C}_{1}, \ldots, \mathcal{C}_{r-1}$ de telle sorte qu'il y ait au moins un entier $n \leq x$ avec $P(n) \leq \sqrt{x / 2}$ qui ne soit pas élément de l'une de ces chaînes. On construit alors une chaîne $\mathcal{C}_{r}$ de la forme suivante :

$$
\mathcal{C}_{r}: p_{2} \mathcal{D}_{r}\left(x / p_{2}, p_{2}\right)-p_{3} \mathcal{D}_{r}\left(x / p_{3}, p_{3}\right)-\ldots-p_{J(x)} \mathcal{D}_{r}\left(x / p_{J(x)}, p_{J(x)}\right)
$$

où $p_{J(x)}$ désigne le plus grand nombre premier $\leq \sqrt{x / 2}$ et où $\mathcal{D}_{r}\left(x / p_{j}, p_{j}\right)$ commence à $2 p_{j-1}$ et finit à 1 .

Pour les $j, 2 \leq j \leq J(x)$, tels que

(6) tous les entiers $n$ vérifiant $n \leq x$ et $P(n)=p_{j}$ sont éléments d'au moins l'une des chaînes $\mathcal{C}_{s}(1 \leq s \leq r-1)$,

on laisse $\mathcal{D}_{r}\left(x / p_{j}, p_{j}\right)$ réduite à ces deux extrémités $2 p_{j-1}$ et 1 . Pour les $j$ ne vérifiant pas $(6)$, on complète $\mathcal{D}_{r}\left(x / p_{j}, p_{j}\right)$ de telle sorte que la sous-chaîne 
$p_{j} \mathcal{D}_{r}\left(x / p_{j}, p_{j}\right)$ contienne au moins un entier $n$ vérifiant $n \leq x$ et $P(n)=p_{j}$, et qui n'apparaît pas dans les chaînes $\mathcal{C}_{s}$ pour $s \leq r-1$. Cela est possible d'après le Lemme 5 , en choisissant 2 comme voisin de $2 p_{j-1}$. On vérifie aisément que la suite $\mathcal{C}_{r}$ ainsi construite est une chaîne d'entiers $\leq x$.

Soit $r=R(x)+1$ le plus petit entier vérifiant pour tout $j, 2 \leq j \leq J(x)$, l'assertion (6). On a alors

$$
R(x) \leq \max _{2 \leq j \leq J(x)} \Psi\left(x / p_{j}, p_{j}\right) \ll x \exp \{-\sqrt{2 \log x}\}
$$

d'après le Lemme 2. Cela achève la démonstration du Lemme 7.

Démonstration du Lemme 8. Bien qu'étant de même nature que celle du Lemme 7, la construction des chaînes prend ici une forme légérement plus complexe. C'est dû à deux phénomènes nouveaux. D'une part, on est ici amené à introduire des connecteurs (notés $a$ ) entre deux sous-chaînes de la forme $p \mathcal{D}(x / p, p)$. D'autre part, on ne peut pas comme précédemment "recouvrir" à l'aide d'une seule chaîne tous les nombres premiers $p_{j}$ vérifiant $\sqrt{x / 2}<p_{j} \leq x / 2^{\alpha_{0}}$. Voyons cela plus en détail.

Pour tout $\alpha$ suffisamment grand, nous allons construire une famille de chaînes $\left(\mathcal{C}_{\alpha, i, j}\right)_{i, j}$ de telle sorte que

(7) tout entier $n$ vérifiant (5) et $\max \left(\sqrt{x / 2}, x / 2^{\alpha+1}\right)<P(n) \leq x / 2^{\alpha}$ soit élément d'au moins l'une des chaînes $\mathcal{C}_{\alpha, i, j}$.

On désigne par $a_{1}<a_{2}<\ldots$ la suite croissante des entiers positifs $a$ vérifiant $\Omega(a) \geq 3$ et on pose

$$
\mathcal{A}_{\alpha}=\left\{8 \leq a \leq 2^{\alpha-2}: \Omega(a) \geq 3\right\} .
$$

Il résulte facilement du théorème des nombres premiers que

$$
\operatorname{card}\{n \leq x: \Omega(n) \leq 2\} \ll \frac{x}{\log x} \log \log x
$$

(voir Théorème II.6.5 de [6] pour un résultat plus général). On peut donc choisir un entier $\alpha_{0}$ suffisamment grand tel que

$$
\operatorname{card} \mathcal{A}_{\alpha} \geq 2^{\alpha-3}-1 \quad \text { pour } \alpha \geq \alpha_{0} .
$$

On désigne par $M(\alpha)$ le plus petit entier tel que

$$
p_{M(\alpha)}>\frac{x}{2^{\alpha+1}} .
$$

Pour $\alpha$ fixé tel que $\alpha \geq \alpha_{0}$ et

$$
2^{\alpha} \leq \sqrt{2 x},
$$

et pour $i$ fixé tel que

$$
i \geq 0 \text { et } M(\alpha)+i 2^{\alpha-3}<M(\alpha-1),
$$

les chaînes $\mathcal{C}_{\alpha, i, j}$ vont être de la forme suivante : 


$$
\begin{aligned}
& p_{M(\alpha)+i 2^{\alpha-3}} \mathcal{D}_{j}\left(x / p_{M(\alpha)+i 2^{\alpha-3}}, p_{M(\alpha)+i 2^{\alpha-3}}\right)-a_{1}- \\
& p_{M(\alpha)+i 2^{\alpha-3}+1} \mathcal{D}_{j}\left(x / p_{M(\alpha)+i 2^{\alpha-3}+1}, p_{M(\alpha)+i 2^{\alpha-3}+1}\right)-a_{2}- \\
& p_{M(\alpha)+i 2^{\alpha-3}+2} \mathcal{D}_{j}\left(x / p_{M(\alpha)+i 2^{\alpha-3}+2}, p_{M(\alpha)+i 2^{\alpha-3}+2}\right)-a_{3}- \\
& \ldots-p_{K(\alpha, i)} \mathcal{D}_{j}\left(x / p_{K(\alpha, i)}, p_{K(\alpha, i)}\right)
\end{aligned}
$$

avec

$$
K(\alpha, i)=\min \left(M(\alpha)+(i+1) 2^{\alpha-3}, M(\alpha-1)\right)-1 .
$$

Soit $a_{l}$ un élément de $\mathcal{A}_{\alpha}$ et $k$ un entier vérifiant

$$
M(\alpha)+i 2^{\alpha-3} \leq k \leq K(\alpha, i) .
$$

D'après (8), (9) et (10), on a $a_{l} \leq \frac{1}{2} \sqrt{x / 2}<p_{M(\alpha)} \leq p_{k}$. Cela entraîne que les connecteurs $a_{l}$ ne sont pas éléments des sous-chaînes $p_{k} \mathcal{D}_{j}\left(x / p_{k}, p_{k}\right)$ de (12).

On suppose construites les chaînes $\left(\mathcal{C}_{\alpha, i, l}\right)$ pour $1 \leq l \leq j-1$. Soit $k$ un entier vérifiant (14) et

$\mathcal{E}_{\alpha, i, j, k}=\left\{n: n\right.$ vérifie $(5), P(n)=p_{k}$ et $n$ n'apparaît dans aucune des chaînes $p_{k} \mathcal{D}_{l}\left(x / p_{k}, p_{k}\right)$ avec $\left.1 \leq l \leq j-1\right\}$.

On utilise alors le Lemme 6 pour montrer que l'on peut effectivement construire des sous-chaînes $p_{k} \mathcal{D}_{j}\left(x / p_{k}, p_{k}\right)$ de telle sorte que $\mathcal{C}_{\alpha, i, j}$ soit une chaîne d'entiers $\leq x$ qui ait la forme (12) et satisfasse la propriété suivante :

$$
\text { pour tout } k \text { tel que } \mathcal{E}_{\alpha, i, j, k} \neq \emptyset \text {, la sous-chaîne } p_{k} \mathcal{D}_{j}\left(x / p_{k}, p_{k}\right) \text { con- }
$$
tient au moins un élément de $\mathcal{E}_{\alpha, i, j, k}$.

C'est avec (8), (9), (13) et (14) que l'on montre que les hypothèses du Lemme 6 sont ici bien vérifiées. On arrête cette construction par récurrence sur $j$ des chaînes $\mathcal{C}_{\alpha, i, j}$, dès que la propriété (7) est vérifiée. On a ainsi construit une famille de chaînes qui recouvre tous les entiers $n$ vérifiant $\sqrt{x / 2}<P(n) \leq x / 2^{\alpha_{0}}$ et $(5)$. Il reste à vérifier que le nombre de ces chaînes est $O(x / \log x)$.

Au regard de la propriété (15), on observe que pour $\alpha$ et $i$ fixés, le nombre de chaînes $\left(\mathcal{C}_{\alpha, i, j}\right)_{j}$ que l'on construit ainsi est

$$
\leq \frac{x}{p_{M(\alpha)+i 2^{\alpha-3}}} \leq \frac{x}{p_{M(\alpha)}}<2^{\alpha+1}
$$

d'après (9). Pour $\alpha$ fixé, le nombre de chaînes $\left(\mathcal{C}_{\alpha, i, j}\right)_{i, j}$ ainsi construites est donc, en utilisant (9) et (11),

$$
<2^{\alpha+1} \operatorname{card}\{i\} \leq 2^{\alpha+1}\left(1+\frac{\pi\left(x / 2^{\alpha}\right)-\pi\left(x / 2^{\alpha+1}\right)}{2^{\alpha-3}}\right) \ll \sqrt{x}+\frac{x}{2^{\alpha} \log x}
$$

d'après (10) et le théorème des nombres premiers. Cela entraîne que le nom- 
bre total des chaînes $\left(\mathcal{C}_{\alpha, i, j}\right)_{\alpha, i, j}$ ainsi construites est $\ll x / \log x$, ce qui achève la démonstration du Lemme 8.

Démonstration du Lemme 9. On a pour $\alpha_{0}$ fixé,

$$
\begin{aligned}
\operatorname{card}\left\{n \leq x: P(n)>x / 2^{\alpha_{0}}\right\} & \leq \sum_{x / 2^{\alpha_{0}<p \leq x}}[x / p] \\
& \leq x \sum_{x / 2^{\alpha_{0}<p \leq x}} 1 / p \ll x / \log x
\end{aligned}
$$

d'après le théorème des nombres premiers. Pour établir le Lemme 9 , il suffit donc de considérer la famille des chaînes réduites à un entier $n$ vérifiant $n \leq x$ et $P(n)>x / 2^{\alpha_{0}}$.

Démonstration du Lemme 10. On désigne par

- $p_{\alpha_{0}}$ le plus petit nombre premier tel que $p_{\alpha_{0}}>x^{4 / 5}$,

- $p_{\alpha_{1}}$ le plus grand nombre premier tel que $p_{\alpha_{1}} \leq x / 2$,

- $p_{\beta_{0}}$ le plus petit nombre premier tel que $p_{\alpha_{0}} p_{\beta_{0}}>x / 2$,

- $p_{\beta_{1}}$ le plus grand nombre premier tel que $p_{\alpha_{0}} p_{\beta_{1}} \leq x$.

Pour un $\alpha$ donné, on désigne par $\beta(\alpha)$ le plus grand entier tel que $p_{\alpha} p_{\beta(\alpha)} \leq x$. On désigne par $\mathcal{C}_{\alpha, \beta}$ la chaîne suivante :

$$
\begin{aligned}
\mathcal{C}_{\alpha, \beta}: p_{\alpha} p_{\beta}-p_{\alpha}- & p_{\alpha} p_{\beta-1}-p_{\beta-1}-p_{\alpha+1} p_{\beta-1}-p_{\alpha+1} \\
& \quad-p_{\alpha+1} p_{\beta-2}-p_{\beta-2}-p_{\alpha+2} p_{\beta-2}-\ldots-p_{\alpha+\beta-1} p_{1} .
\end{aligned}
$$

On considère les chaînes $\mathcal{C}_{\alpha, \beta}$ avec $\left[\alpha=\alpha_{0}\right.$ et $\left.\beta_{0} \leq \beta \leq \beta_{1}\right]$ ou $\left[\alpha_{0} \leq\right.$ $\alpha \leq \alpha_{1}$ et $\left.\beta=\beta(\alpha)\right]$. D'après le Lemme 3 , on a pour $p_{i}>x^{4 / 5}, p_{j}<x^{1 / 5}$ et $x$ suffisamment grand,

$$
p_{i+1} p_{j-1}<p_{i} p_{j} .
$$

Cela montre que les chaînes $\mathcal{C}_{\alpha, \beta}$ considérées sont formées d'entiers $\leq x$. Par ailleurs, d'une part ces chaînes $\mathcal{C}_{\alpha, \beta}$ recouvrent tous les entiers $n$ tels que $x / 2<n \leq x, \Omega(n)=2$ et $P(n)>x^{4 / 5}$. D'autre part, le nombre de ces chaînes est, d'après le théorème des nombres premiers, $O(x / \log x)$. Enfin, on a

$$
\begin{aligned}
\operatorname{card}\{x / 2<n \leq & \left.x: \Omega(n)=2 \text { et } P(n) \leq x^{4 / 5}\right\} \\
\leq & \sum_{\substack{p, q \\
x^{1 / 5} / 2<p \leq q \leq x / p}} 1 \leq \sum_{x^{1 / 5} / 2<p \leq \sqrt{x}} \pi(x / p) \asymp x / \log x,
\end{aligned}
$$

d'après le théorème des nombres premiers. Cela permet de compléter les chaînes $\mathcal{C}_{\alpha, \beta}$ par des chaînes réduites à un élément et ainsi d'achever la démonstration du Lemme 10. 


\section{Bibliographie}

[1] P. Erdős, R. Freud and N. Hegyvári, Arithmetical properties of permutations of integers, Acta Math. Hungar. 41 (1983), 169-176.

[2] M. N. Huxley, The Distribution of Prime Numbers, Clarendon Press, Oxford, 1972.

[3] A. D. Pollington, There is a long path in the divisor graph, Ars. Combin. 16-B (1983), 303-304.

[4] C. Pomerance, On the longest simple path in the divisor graph, Congr. Numer. 40 (1983), 260-268.

[5] E. Saias, Applications des entiers à diviseurs denses, prépublication.

[6] G. Tenenbaum, Introduction à la théorie analytique et probabiliste des nombres, Inst. Élie Cartan 13, Université de Nancy I, 1990.

[7] —, Sur un problème de crible et ses applications, 2. Corrigendum et étude de graphe divisoriel, Ann. Sci. École Norm. Sup. (4) 28 (1995), 115-127.

MATHEMATICAL INSTITUTE

HUNGARIAN ACADEMY OF SCIENCES

REÁLTANODA U. 13-15

BUDAPEST, H-1053 HUNGARY
LABORATOIRE DE PROBABILITÉS UNIVERSITÉ PARIS VI

4, PLACE JUSSIEU 